\title{
Supercritical fluid impregnation of a biocompatible polymer for ophthalmic drug delivery
}

\author{
Ana Rita C. Duarte ${ }^{\mathrm{a}, *}$, Ana Luisa Simplicio ${ }^{\mathrm{b}}$, Arlette Vega-González ${ }^{\mathrm{c}}$, Pascale Subra-Paternault ${ }^{\mathrm{c}}$, \\ Patrícia Coimbra $^{\mathrm{d}}$, M.H. Gil ${ }^{\mathrm{d}}$, Herminio C. de Sousa ${ }^{\mathrm{d}}$, Catarina M.M. Duarte ${ }^{\mathrm{a}}$ \\ a Nutraceuticals and Delivery Laboratory, ITQB/IBET, Aptd. 12, 2780 Oeiras, Portugal \\ ${ }^{\mathrm{b}}$ Pharmacokinetics and Biopharm. Analysis Laboratory, ITQB/IBET, Aptd. 12, 2780 Oeiras, Portugal \\ ${ }^{\mathrm{c}}$ Laboratoire d'Ingénierie des Matériaux et des Hautes Pressions, C.N.R.S., Institut Galilée, Université 13, \\ 99 Avenue Jean Baptiste Clément, 93430 Villetaneuse, France \\ ${ }^{\mathrm{d}}$ Departamento de Engenharia Química, FCT, Universidade de Coimbra, Pólo II-Pinhal de Marrocos, Rua Sílvio Lima, 3030-790 Coimbra, Portugal \\ Received 22 September 2006; received in revised form 28 January 2007; accepted 28 January 2007
}

\begin{abstract}
Supercritical fluid impregnation was tested to prepare a new ophthalmic drug delivery device. Poly(methylmethacrylate-co-ethylhexylacrylateco-ethyleneglycoldimethacrylate), P(MMA-EHA-EGDMA) has been proposed by Mariz [M. Mariz, Preparação de uma lente intra-ocular dotada de um sistema de libertação controlada de fármaco, Master Thesis, Universidade de Coimbra, 1999] as a promising matrix to be used for intraocular delivery of anti-inflammatory drugs used in eye surgery. This matrix was successfully impregnated with flurbiprofen, a non-steroidal anti-inflammatory agent.

The success of the impregnation was evaluated by scanning electron microscopy (SEM) analysis and also by in vitro drug release studies. The effect of some operating parameters was evaluated, namely, pressure and contact time. The operating pressure will influence both the solubility of the drug in the supercritical fluid but also the sorption degree of the polymeric matrix in the presence of carbon dioxide. The solubility of the drug in carbon dioxide and the sorption degree are reported in previous studies. A comparison between the batch and the semi-continuous impregnation process is also presented.

The supercritical fluid impregnation proved to be feasible for the preparation of a new ophthalmic drug delivery system. The drug release profiles suggest that the drug can be released up to three months, which is a major advantage for the prevention of the inflammatory response after ophthalmic surgery.
\end{abstract}

(C) 2007 Elsevier B.V. All rights reserved.

Keywords: Supercritical fluids; Impregnation; Biocompatible polymers; Ophthalmic drug delivery

\section{Introduction}

Cataract is the most common treatable form of blindness worldwide and the most frequent form of ophthalmic surgical procedure is to extract the cataract with subsequent implant of an intra-ocular lens (IOL) to compensate the loss of the natural crystalline lens. Poly(methylmethacrylate-co-ethylhexylacrylateco-ethyleneglycoldimethacrylate), P(MMA-EHA-EGDMA) has been proposed by Mariz [1] as a promising matrix to be used for intraocular delivery of anti-inflammatory drugs used in eye surgery. The inflammatory response of the ocular tissues

\footnotetext{
* Corresponding author. Tel.: +35121446 9728.

E-mail address: rduarte@itqb.unl.pt (A.R.C. Duarte).
}

in a common-side effect associated to ophthalmic surgery. It can interfere with the normal function of the eye, whose optic transparency must be maintained [2]. Most ocular diseases are treated with optical application of drug solutions administered as eye drops. These medications present several problems and/or limitations, like poor drug bioavailability and systemic toxicity, derived essentially from two main features: ocular anatomical and physiological constraints, and pharmacokinetic and other carrier limitations. Poor ocular drug bioavailability is due essentially to the relative impermeability of the corneal membrane, to tear dynamics, blinking and nasolacrimal drainage, and to the high efficiency of the blood-ocular barrier. In the case of eye drops medication, only $5 \%$ of the applied drug actually penetrates the cornea [3]. The initial high drug concentration present in tears, followed by its rapid decline, 
due mainly to drainage, usually forces clinicians to recommend frequent drug dosing, at high concentrations, in order to avoid poor drug bioavailability but causing a potential risk of systemic toxicity. Therefore, topical eye drops are an ineffective delivery system, with an active principle concentration of 20 times higher than the effectively needed; some of the drug not absorbed by the cornea will reach the bloodstream, as tear dynamics and nasolacrimal duct drainage are also a major way of entry into the circulatory system which may cause undesired and toxic systemic side-effects. In most cases, the delivery of ocular drugs to the posterior segment of the eye is diminished by the same factors that are responsible for the poor ocular bioavailability and also by the blood-retinal barrier which limits the intravenous route in posterior drug delivery [4-6]. However, topical eye drops accounts for almost $90 \%$ of the commercially available therapeutic ophthalmic products because of its favorable costs, simplicity of formulation and self-application, and good acceptance by patients, despite the referred disadvantages and related risks. Several efforts have been made in order to improve the ocular delivery of topically applied ocular drugs and to reduce their disadvantages and adverse effects $[4,6]$.

Controlled drug delivery products, using biocompatible or biodegradable polymers, have received considerable attention in the last years. These substances are generally used for the preparation of controlled release formulations, which, therefore allow to control the rate of assumption of the drug by the body improving its pharmacokinetic profile. In fact, there is a growing interest of the pharmaceutical industry in the development of these systems [7].

The preparation of drug release products necessitates the use of a mobile phase that dissolves and carries the drug component, which also swells and stretches the polymer matrix, facilitating the diffusion of the drug and increasing the rate of impregnation. Conventionally, the preparation of these systems involves three steps: solubilization of the pharmaceutical in an appropriate solvent, diffusion of the pharmaceutical through the polymer and elimination of the residual solvent.

Impregnation using supercritical fluid technology has proven to be feasible when the pharmaceutical compound is soluble in carbon dioxide and the polymer can be swollen by the supercritical fluid. A high purity product, free of residual solvents is obtained, since no organic solvents are involved in the impregnation process [8,9]. Supercritical fluids, especially supercritical carbon dioxide $\left(\mathrm{scCO}_{2}\right)$ have been identified as prime candidates to develop alternative clean processes for the preparation of drug-loaded polymeric matrixes [10]. Furthermore, the use of supercritical fluids can take advantage of their high diffusivity in polymers, in combination with the high solubility and plasticizing action. Therefore, compressed $\mathrm{CO}_{2}$ can be used as a solvent to carry the drug into biocompatible polymers. Moreover, when depressurisation occurs, the gas rapidly diffuses out of the polymer, deplasticizing it and warranting the complete removal of solvent, without exposing polymers and drugs to high temperatures, which may degrade them [11,12].

In this work, the possibility of impregnating P(MMA-EHAEGDMA) with flurbiprofen was evaluated. The solubility of the drug in carbon dioxide had been previously studied, as well as the sorption degree of this polymeric matrix in the presence of carbon dioxide. The aim of this study was to evaluate the effects of these two variables in the impregnation process, in order to determine the best operating conditions. The percentage of drug impregnated in the films as well as the release profiles of the drug out of the film were evaluated and the results are presented.

\section{Experimental procedure}

\subsection{Materials}

P(MMA-EHA-EGDMA) samples were synthesized by bulk free radical polymerization, and according to the method described below [1]. Flurbiprofen, (2-fluoro-alpha-methyl-4biphenylacetic acid), CAS [5104-49-4], (97\% purity) was purchased from Sigma-Aldrich. Carbon dioxide (99.5\%, industrial grade) was obtained from Air Liquide (France). All products were used with no further purification.

\subsection{Polymerization}

Reaction mixtures were prepared weighing the liquid monomers and the thermal initiator. The reticulated copolymer P(MMA-EHA-EGDMA) was prepared adding $40 \%$ of MMA, $60 \%$ EHA and $7.5 \%$ (weight \% of total mass of MMA + EHA) of the reticulating monomer, EGDMA. Benzoyl peroxide was used as initiator with $1 \%$ concentration (molar $\%$ of total moles of MMA + EHA). MMA's stabilizer was previously removed by liquid-liquid extraction.

Reaction mixtures were magnetically stirred for $10 \mathrm{~min}$ until benzoyl peroxide was completely dissolved. Then, the resulting mixtures were spread between glass plates, separated with silicone rubber spacers, in order to produce fine polymer films of uniform thickness $(\sim 1 \mathrm{~mm})$. Polymerization reactions were carried out for three hours, in a heated oven, at $80{ }^{\circ} \mathrm{C}$. The obtained samples were cut in circular shape samples ( $9.0 \mathrm{~mm}$ diameter). All samples were vacuum dried and weighted, until constant mass is achieved (in order to remove any possible monomer residue still present).

\subsection{Supercritical fluid impregnation process}

The procedure is similar to the one described by Diankov [13] and the impregnation apparatus is schematically presented in Fig. 1.

The equipment consists basically in a saturator, $\mathrm{S}$, an impregnation vessel, I. S and I are tubular vessels (of 5 and $10 \mathrm{~mL}$, respectively) placed in an air oven (Touzard et Matignon, model WTE Binder) that maintains the temperature within $\pm 1{ }^{\circ} \mathrm{C}$. The fluid temperature is measured by means of a thermocouple (WATLOW, 965 series). $\mathrm{CO}_{2}$ was liquefied through a cooling unit CU (Colora, model WK15), and compressed to the operating pressure with a pump (Gilson, 308). The fluid was preheated to the desired temperature in a heat exchanger, HE, before entering the saturator. The pressure of the system is measured with a pressure transducer inline with the saturator 


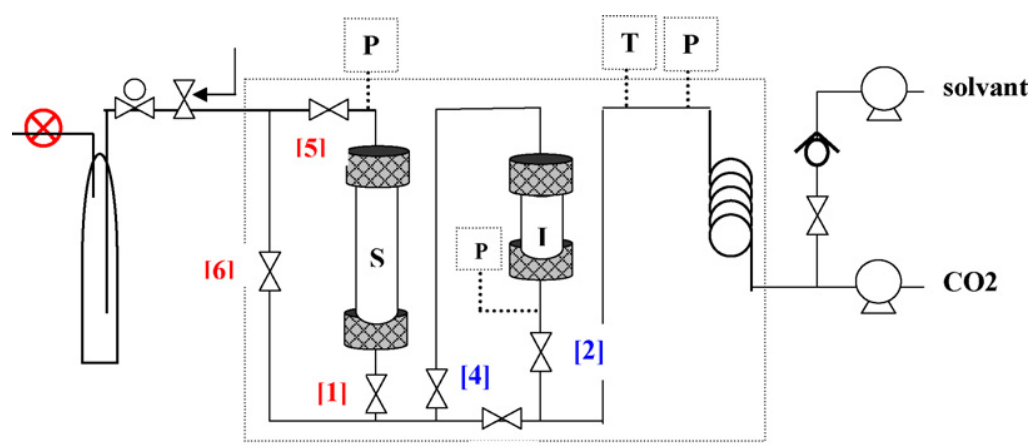

[3]

Fig. 1. Supercritical fluid impregnation apparatus, (S) saturator, (I) impregnation vessel, $(\mathrm{P})$ pressure transducer, $(\mathrm{T})$ temperature sensor.

and the impregnation vessel (ASCO, model PR711F). The saturator was loaded with a few grams of pharmaceutical drug and small glass beads to enhance the mass transfer and the impregnation vessel was loaded with the polymer. A typical impregnation experiment started by stabilizing the system with $\mathrm{CO}_{2}$ during a period of $2 \mathrm{~h}$, to achieve the maximum sorption degree of the polymer, and also to promote the solubilization of the drug in carbon dioxide. Valves 1,2 and 3 were kept open and 4, 5 and 6 closed, during the swelling period. Then, the $\mathrm{CO}_{2}$ was allowed to flow through the vessels for a selected period of time. Valves 1, 2, 4 and 5 were opened and 3 was closed during the impregnation step. The carbon dioxide flow was maintained constant for all experiments performed $(\sim 1$ SLPM) and it was controlled by means of a metering valve. The flow was measured by a flow meter (Kobold, model MAS 4009). At the end of the flow period, the system was slowly depressurized.

The batch impregnation process was carried out in the same apparatus but in this case both, the polymer and the drug were loaded in the same vessel. Flurbiprofen was loaded in the bottom of the cell and it was separated from the polymeric matrixes with a cotton layer in order to prevent contact of the two and thus, the contamination of the surface of the films with the powder. The vessel was set at the desired operational conditions ( $T$ and $P$ ) and the impregnation took place for the pre-determined period.

\subsection{Film characterization}

\subsubsection{Scanning electron microscopy (SEM)}

The morphology of polymer samples was analysed and imaged by scanning electron microscopy (SEM, Leica 5440) after carbon sputter coating.

\subsubsection{In vitro release studies}

Polymeric impregnated films were suspended in $40 \mathrm{~mL}$ of simulated lacrimal fluid (prepared according to USPharmacopea 26) stirred at $150 \mathrm{rpm}$ at $37^{\circ} \mathrm{C}$. Aliquots of $500 \mu \mathrm{L}$ were withdrawn in predetermined time intervals and the same volume of fresh medium was added to the suspension. The samples were filtered $(0.45 \mathrm{~mm}$, milipore filters) and analysed by HPLC (Merck Hitachi consisting of a L-6200 pump, A-2000 autosampler, L-4200 UV-vis detector and a T-6300 column thermostat). A C8 column (Merck, Lichrospher 250-4, $5 \mu \mathrm{m}$ ) and a precolumn, kept at $40^{\circ} \mathrm{C}$, was used in isocratic conditions with a mobile phase consisting of $1.4 \mathrm{~g}$ of monobasic sodium phosphate monohydrate (p.a. Panreac) in $570 \mathrm{~mL}$ of deionised water and $430 \mathrm{~mL}$ of acetonitrile (HPLC grade, Lab-Scan) adjusted to pH 3 with phosphoric acid (85\% Panreac). The flow rate was $1.5 \mathrm{~mL} / \mathrm{min}$ and the volume injected $50 \mu \mathrm{L}$. Detection was at $254 \mathrm{~nm}$ and the quantification was by external standard calibration. The retention time of flurbiprofen under these conditions was 8.3 min. Calculations of the amount of drug released took into account replacement of aliquots with fresh medium.

\section{Results}

The characterization of the binary systems drug- $-\mathrm{CO}_{2}$ and $\mathrm{CO}_{2}$-polymer is essential for the determination of the best operating conditions. Solubility data of flurbiprofen is reported previously [14] as well as the sorption of the carbon dioxide in the polymeric matrix $[15,16]$. Drug solubility is defined as the amount of solute (drug) that can be dissolved in a certain amount of solvent (supercritical fluid) at certain operational conditions (pressure and temperature). The sorption degree was defined to be the ratio between the mass of carbon dioxide in the sample and total mass (mass of polymeric sample + mass of carbon dioxide). The solubility of flurbiprofen in $\mathrm{scCO}_{2}$ at the operational conditions tested and the sorption of carbon dioxide in $\mathrm{P}$ (MMA-EHA-EGDMA) are reported in Table 1.

Impregnation experiments were performed either in a batch or semi-continuous mode in order to evaluate the performance of these two techniques. The experiments were carried out at pressures ranging from 100 to 180 bar and temperatures from 308 to $313 \mathrm{~K}$. Also, different contact times during the impregnation process were studied (Table 1).

The resulting impregnated polymer films were cut into sections and characterized using SEM. Two examples of impregnated polymers are presented in Fig. 2(a and b).

As it can be seen from the SEM images, the drug is more homogeneously dispersed within the matrix when the batch process was performed.

Furthermore, the sample processed in batch mode presents a higher impregnation yield, than the one prepared using a semi-continuous method. The percentage of impregnation is defined as the relative quantity of dug in an impregnated sample, expressed in $\mathrm{w} / \mathrm{w}$ percentage. It is probable that the flow rate of $2 \mathrm{~mL} / \mathrm{min}$ is too high to get a contact time long enough for 
Table 1

Summary of the operational conditions of the experiments performed

\begin{tabular}{llllllll}
\hline \# Experiment & $P($ bar) & $T\left({ }^{\circ} \mathrm{C}\right)$ & $\begin{array}{l}\mathrm{CO}_{2} \text { flow } \\
(\mathrm{mL} / \mathrm{min})\end{array}$ & $\begin{array}{l}\text { Impregnation } \\
\text { time }(\mathrm{h})\end{array}$ & $\begin{array}{l}\text { Sorption degree } \\
(\mathrm{wt} \%)\end{array}$ & $\begin{array}{l}\text { Drug solubility } \\
(\mathrm{g} / \mathrm{L})\end{array}$ \\
\hline 1 & & 2 & 3 & 60 & 0.34 & 0.22 \\
2 & 180 & 35 & 2 & 3 & 57 & 0.26 & 0.11 \\
3 & 150 & 35 & 2 & 3 & 69 & 0.34 & 0.37 \\
4 & 100 & 35 & 2 & 5 & 60 & 0.34 \\
5 & 180 & 35 & 35 & 3 & 0.32 \\
\hline
\end{tabular}
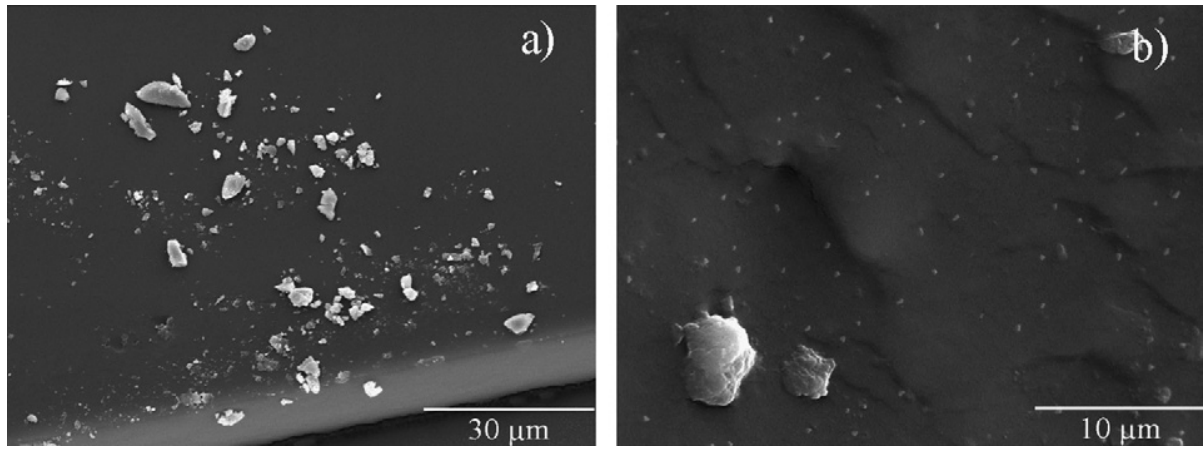

Fig. 2. SEM images of experiments (a) 1 and (b) 5.

the concentration in the fluid phase to approach the equilibrium value. Thus, the poor mass transfer of dissolution and impregnation due to this too high flow rate does not ensure a contact time equivalent to the one in the batch process.

Another parameter that greatly influences the amount of drug impregnated is the contact time. Increasing the impregnation time, there is also an increase in the percentage of drug impregnated in the polymeric matrix. However, when the batch process was performed better impregnation yields were achieved, even at a shorter contact time. The high carbon dioxide flow, in the semi-continuous process can be one of the reasons for the results obtained (Table 1).

In Fig. 3, the effect of pressure in the impregnation yield can be evaluated. Comparing the percentage of impregnation of the experiments performed at different pressures it can be concluded that at higher pressures lead to a lower percent of impregnation. This represents also the trend of the percentage of impregnation versus the sorption degree of the polymer. A lower sorption degree favours the process, therefore lower operational pressures should be used for higher impregnation yields.

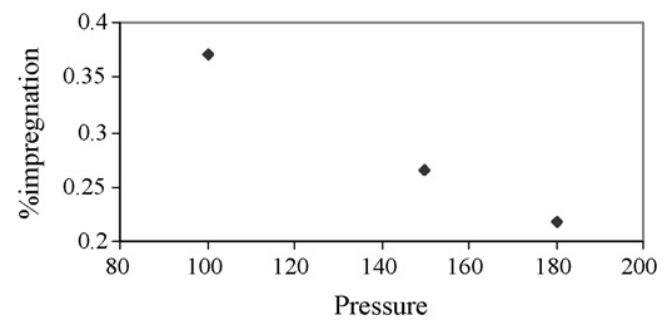

Fig. 3. Percentage of impregnation vs. operational pressure.
Kazarian et al. [12,17,18] distinguish two mechanisms of impregnation assisted by supercritical fluids. The first mechanism corresponds to a simple deposition of the compound when the fluid leaves the swollen matrix. In this case, the solute is solubilized in carbon dioxide and the polymer is exposed to this solution for a predetermined period followed by depressurization of the system. When the system is depressurised, carbon dioxide molecules quickly leave the polymer matrix, leaving the solute trapped inside. As reported by Kazarian, it concerns mostly solute with a relatively high solubility in the fluid and it is specific to impregnation carried out on a matrix subjected to swelling upon exposure to supercritical fluid. The second effect, not specific to supercritical fluids, corresponds to chemical interactions (like van der Waal's interactions) between the solute and the matrix, that would favour the preferential partitioning of the solute with the polymer phase.

Flurbiprofen has a rather high solubility in carbon dioxide and it increases with pressure. Additionally, the sorption of carbon dioxide in the matrix also increases with pressure for a certain temperature. So it would be expected an increase of the solute uptake with pressure, on the basis that the more the $\mathrm{CO}_{2}$ is loaded with the drug and the easier it goes inside the polymer, the larger should be the drug uptake. But, it is the contrary that is observed. Indeed, the experiments show that these two effects when combined do not favour the impregnation process. This might be due to stronger interactions of the drug for the solvent (carbon dioxide) than for the polymeric matrix that is poorly impregnated. As reported in previous works by Subra-Paternault and co-workers [19,20] at higher densities (or higher temperatures), the interactions between the solute and the carbon dioxide molecules increase and are detrimental to the bonding forces between the solute and the matrix, therefore 


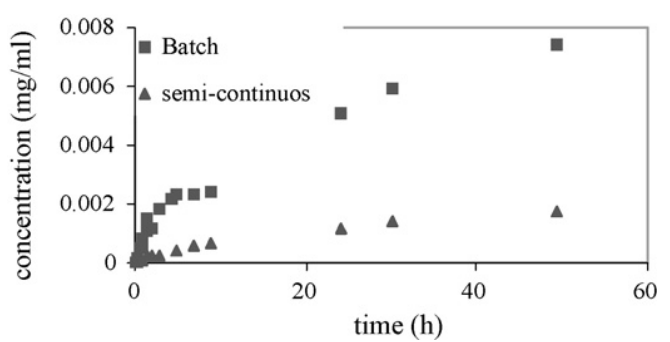

Fig. 4. Release profiles of the impregnated samples (experiments 1 and 5).

at higher pressures and temperatures the impregnation yield is lower.

The drug release profiles were similar for all the impregnated samples and an example is given in Fig. 4.

The release profiles of the samples prepared prove the success of the impregnation process and indeed, P(MMA-EHAEGDMA) might be used as a new ophthalmic drug delivery device. It is also relevant the fact that flurbiprofen is still being released up to more than three months. This can present an enormous advantage for the prevention of the inflammatory response after ophthalmic surgery.

\section{Conclusions}

P(MMA-EHA-EGDMA) was successfully impregnated with flurbiprofen, an anti-inflammatory agent. Different experimental conditions were tested and the results obtained suggest that the best impregnating conditions for this system are low temperatures and pressures, which at the same time correspond to a lower solubility of the drug in the supercritical fluid and a low swelling of the polymeric matrix.

Impregnation efficiency results from a complex mechanism that, in turn, involves interactions between the solute, the carrier and the matrix. The relative strength of all binary interactions will contribute to the final partitioning of the solute between the mobile phase and the solid. In the case of the polymer and drug studied in this work, results suggest that the interactions between the drug and the fluid phase are stronger than the ones of the drug to the polymeric matrix, therefore lower solvent densities favour the impregnation process.

Experiments performed also indicate that the batch impregnation process leads to higher yields of impregnation and according to the release profiles obtained the drug can be released from the matrix up to three months, which presents great advantages for post-surgical treatments.

\section{Acknowledgments}

Ana Rita C. Duarte is grateful for financial support from SFRH/BD/10780/2002 grant. Authors gratefully acknowledge the support of French-Portuguese PAUILF 2005 program. This work was also partially supported by FCT-MCES and FEDER, Portugal, under contract POCTI/FCB/38213/2001.

\section{References}

[1] M. Mariz, Preparação de uma lente intra-ocular dotada de um sistema de libertação controlada de fármaco, Master Thesis, Universidade de Coimbra, 1999.

[2] R. Pignatello, C. Bucolo, G. Spedalieri, A. Maltese, G. Puglisi, Flurbiprofen-loaded acrylate polymer nanosuspensions for ophthalmic application, Biomaterials 23 (2002) 3247-3255.

[3] A.F. Clark, T. Yorio, Ophthalmic drug discovery, Nat. Rev. Drug Discov. 2 (2003) 448-459.

[4] H. Sasaki, K. Yamamura, K. Nishida, J. Nakamura, M. Ichikawa, Delivery of drugs to the eye by topical application, Prog. Retin. Eye Res. 15 (1996) 583-619.

[5] S. Ding, Recent developments in ophthalmic drug delivery, Pharm. Sci. Tech. Today 1 (1998) 328-335.

[6] C. Le Bourlais, L. Acar, H. Zia, P.A. Sado, T. Needham, R. Leverge, Ophthalmic drug delivery systems—recent advances, Prog. Retin. Eye Res. 17 (1998) 33-58.

[7] J. Heller, in: B.D. Ratner, A.S. Hoffmann, F.J. Schoen, J.E. Lemons (Eds.), Drug Delivery Systems, Biomaterials Science, An Introduction to Materials in Medicine, Academic Press, London, UK, 1996, pp. 346-356.

[8] I. Kikic, F. Vecchione, Supercritical impregnation of polymers, Curr. Opin. Solid State Mater. Sci. 7 (2003) 399-405.

[9] C. Elvira, A. Fanovich, M. Fernández, J. Fraile, J.S. Román, C. Domingo, Evaluation of drug delivery characteristics of microspheres of PMMAPCL-Cholesterol obtained by supercritical $\mathrm{CO}_{2}$ impregnation and by dissolution-evaporation techniques, J. Controll. Rel. 99 (2004) 231-240.

[10] I. Kikic, P. Sist, Applications of supercritical fluids to pharmaceuticals: controlled drug delivery systems, supercritical fluids: fundamentals and applications, in: Proceedings of the Second NATO ASI on Supercritical Fluids, NATO Science Series, Kluwer Academic Publishers, Dordrecht, The Netherlands, 2000, pp. 291-306.

[11] A.R. Berens, G.S. Huvard, R.W. Korsmeyer, F.W. Kunig, Application of compressed carbon dioxide in the incorporation of additives into polymers, J. Appl. Polym. Sci. 46 (1992) 231-242.

[12] S.G. Kazarian, Supercritical Fluid Impregnation of Polymers for Drug Delivery, Supercritical Fluid Technology for Drug Product Development, Marcel Dekker, Inc., 2004, p. 343.

[13] S. Diankov, Etude experimentale et modélisation d'une imprégnation en milieu $\mathrm{CO}_{2}$ supercritique: équilibre et cinétique, Ph.D. Thesis, Universite Paris 13, France, 12 mai 2005.

[14] A.R.C. Duarte, P. Coimbra, H. de Sousa, C.M.M. Duarte, Solubility of flurbiprofen in supercritical carbon dioxide, J. Chem. Eng. Data 49 (3) (2004) 449-452.

[15] A.R.C. Duarte, C. Martins, P. Coimbra, M.H.M. Gil, H.C. de Sousa, C.M.M. Duarte, Sorption and diffusion of supercritical carbon dioxide in a biocompatible polymer, J. Supercrit. Fluids (Available online, Janeiro 2006).

[16] A.R.C. Duarte, L. Anderson, C.M.M. Duarte, S.G. Kazarian, A comparison between gravimetric and in-situ spectroscopic methods to measure the sorption of $\mathrm{CO}_{2}$ in a biocompatible polymer, J. Supercrit. Fluids 36 (2005) $160-165$.

[17] S.G. Kazarian, Polymer processing with supercritical fuids, Polym. Sci. Ser. C 42 (1) (2000) 78-101.

[18] S.G. Kazarian, G.G. Martirosyan, Spectroscopy of polymer/drug formulations processed with supercritical fluids: in situ ATR-IR and Raman study impregnation of ibuprofen into PVP, Int. J. Pharm. 232 (2002) 8190.

[19] P. Subra-Paternault, A. Vega-Bancel, E. Reverchon, Breakthrough curves and adsorption isotherms of terpene mixtures in supercritical carbon dioxide, J. Supercrit. Fluids 12 (1998) 43-57.

[20] S. Diankov, D. Barth, A. Vega-González, I. Pentchev, P. Subra-Paternault, Impregnation isotherms of hydroxybenzoic acid on PMMA in supercritical carbon dioxide, J. Supercrit. Fluids 41 (2007) 164-172. 